\title{
Modelling Wave-current Interaction and Its Potential Influence on the Transport of Materials in Lake Taihu, China
}

\author{
Bo Yao, a , Qingquan Liü, b \\ ${ }^{1}$ Key Laboratory for Mechanics in Fluid Solid Coupling System, Institute of Mechanics, Chinese \\ Academy of Sciences, Beijing 100190, China \\ ayaobo@imech.ac.cn, bqqliu@imech.ac.cn
}

Keywords: wave; current; interaction; transport; Lake Taihu.

Abstract. A wave-current coupled model was established to investigate the interaction of wind-induced wave and current and its potential effect on the transport of materials in Lake Taihu, China. Different wind conditions were considered, and numerical experiments on the transportation of dissolved materials and particles were carried out in the northern part of Meiliang Bay. The results showed that the influences between wave and current were spatially heterogeneous. And under the given conditions, wave had an influence of less than about $5 \%$ on the current speed, but current could lead to more than $10 \%$ difference of the computed significant wave height. And the wave-current interaction was found to have larger impact on particles than on dissolved substances in the surface water, suggesting that this kind of interaction could be neglected when simulating the transportation of dissolved substances such as nitrogen and phosphorus, but may need to be considered in the studying of buoyant algae when its particle characteristic is important.

\section{Introduction}

Wind induced wave and current are two important aspects of hydrodynamic process in large shallow lakes, which to some extent determine the fate of materials such as nutrients, sediments, and algae in the water. The interaction between waves and current flows also attract great attentions of researchers and engineers, as it also affect the transporting process in the waterbody.

Lake Taihu is a large, shallow, eutrophic lake in the Yangtze Delta of China. Fast increase in the amount of sewage discharged into the lake since the 1980s leads to high loads of nutrients in the water, and heavy algal blooms mainly caused by Microcystis spp. occurred frequently in the recent decades ${ }^{[1]}$. Hydrodynamic condition plays important roles in the transportation of nutrients and algal mass in the water, and has caused increasing interests in the recent decades.

Previous researches ${ }^{[2,3]}$ on hydrodynamics in Lake Taihu mainly focused on either wind-induced current or wave. Few studies reported the interaction between them and its impact on the transport of nutrients and algal mass in the lake. In this paper, a three dimensional current model and a wave model were coupled to study the basic characteristics of this interaction and its potential influences on the transport of substances in the water.

\section{Materials and Methods}

Model Descriptions. Wind-induced current was simulated by MIKE3 FM, which is a software package for three dimensional hydrodynamic computations from the DHI Company. The model is based on the solution of the three-dimensional incompressible Reynolds averaged Navier-Stokes equations, subject to the assumptions of Boussinesq and of hydrostatic pressure. The local continuity equation is written as ${ }^{[4]}$ :

$$
\frac{\partial u}{\partial x}+\frac{\partial v}{\partial y}+\frac{\partial w}{\partial z}=S
$$

And the two horizontal momentum equations for the $\mathrm{x}$ - and $\mathrm{y}$-component are as: 


$$
\begin{aligned}
& \frac{\partial u}{\partial t}+\frac{\partial u^{2}}{\partial x}+\frac{\partial v u}{\partial y}+\frac{\partial w u}{\partial z}=f v-g \frac{\partial \eta}{\partial x}-\frac{1}{\rho_{0}} \frac{\partial p_{a}}{\partial x}-\frac{g}{\rho_{0}} \int_{z}^{\eta} \frac{\partial \rho}{\partial x} d z-\frac{1}{\rho_{0} h}\left(\frac{\partial s_{x x}}{\partial x}+\frac{\partial s_{x x}}{\partial y}\right)+F u+\frac{\partial}{\partial z}\left(v_{t} \frac{\partial u}{\partial z}\right)+u_{s} S \\
& \frac{\partial v}{\partial t}+\frac{\partial v^{2}}{\partial x}+\frac{\partial u v}{\partial y}+\frac{\partial w v}{\partial z}=f u-g \frac{\partial \eta}{\partial y}-\frac{1}{\rho_{0}} \frac{\partial p_{a}}{\partial y}-\frac{g}{\rho_{0}} \int_{z}^{\eta} \frac{\partial \rho}{\partial y} d z-\frac{1}{\rho_{0} h}\left(\frac{\partial s_{y x}}{\partial x}+\frac{\partial s_{y y}}{\partial y}\right)+F v+\frac{\partial}{\partial z}\left(v_{t} \frac{\partial v}{\partial z}\right)+v_{s} S
\end{aligned}
$$

Where $t$ is the time; $x, y$, and $z$ are the Cartesian coordinates; $u, v$, and $w$ are the velocity components in the $x, y$, and $z$ direction; $\eta$ is the surface elevation; $d$ is the still water depth; $h$ is the total water depth; $f$ is the Coriolis parameter; $\rho$ is the water density; $S_{x x}, S_{x y}$, and $S_{y x}$ and $S_{y y}$ are the components of the radiation stress tensor; $v_{t}$ is the vertical turbulent viscosity; $P_{\mathrm{a}}$ is the atmospheric pressure; $S$ is the magnitude of the discharge due to point sources.

The main equation of the wave model can be written as:

$$
\frac{\partial}{\partial t} N+\frac{\partial}{\partial x} C_{x} N+\frac{\partial}{\partial y} C_{y} N+\frac{\partial}{\partial \sigma} C_{\sigma} N+\frac{\partial}{\partial \theta} C_{\theta} N=\frac{S}{\sigma}
$$

where $N(\sigma, \theta)$ represents the action density spectrum; $C_{x}, C_{y}, C_{\sigma}$, and $C_{\theta}$ are spreading velocities in the directions of $x, y, \sigma$, and $\theta ; S$ is a source term. The current and wave models were coupled at each time step, in which wave radiation stress $\left(S_{x x}, S_{y y}, S_{x y}\right)$ was passed from the wave model to the current model, and current speed $(u, v$, and $w)$ from the current model to the wave model.

The transport of dissolved substance can be described by the advection-diffusion equation:

$$
\frac{\partial C}{\partial t}+\frac{\partial u C}{\partial x}+\frac{\partial v C}{\partial y}+\frac{\partial w C}{\partial z}=F_{c}+\frac{\partial}{\partial z}\left(D_{v} \frac{\partial C}{\partial z}\right)-k_{\mathrm{p}} C+C_{\mathrm{s}} S
$$

where $C$ is the concentration, $K_{\mathrm{p}}$ is a degradation coefficient, $S$ is a source term.

The transport and dispersion of particles follows the Langevin equation which could be written as:

$$
d X t=a\left(t, X_{t}\right) d t+b\left(t, X_{t}\right) \xi_{t} d t
$$

Where $a$ is a drift term, $b$ is a diffusion term, and $\xi$ is a random number.

Model Setup. The horizontal mesh (shown in Fig.1, left) for the current model was consisted of 3127 unstructured triangular elements, and there were 5 equidistant $\sigma$ layers in the vertical direction. The total number of elements is 15635 . The wave model shared the same horizontal mesh of the current model. A point source was set at the northern end of the lake to carry out numerical transport experiments of both dissolved materials and particles. And three probes (P1, P2, and P3) were set to examine the concentrations of the materials in the numeric experiments (Fig.1, right).

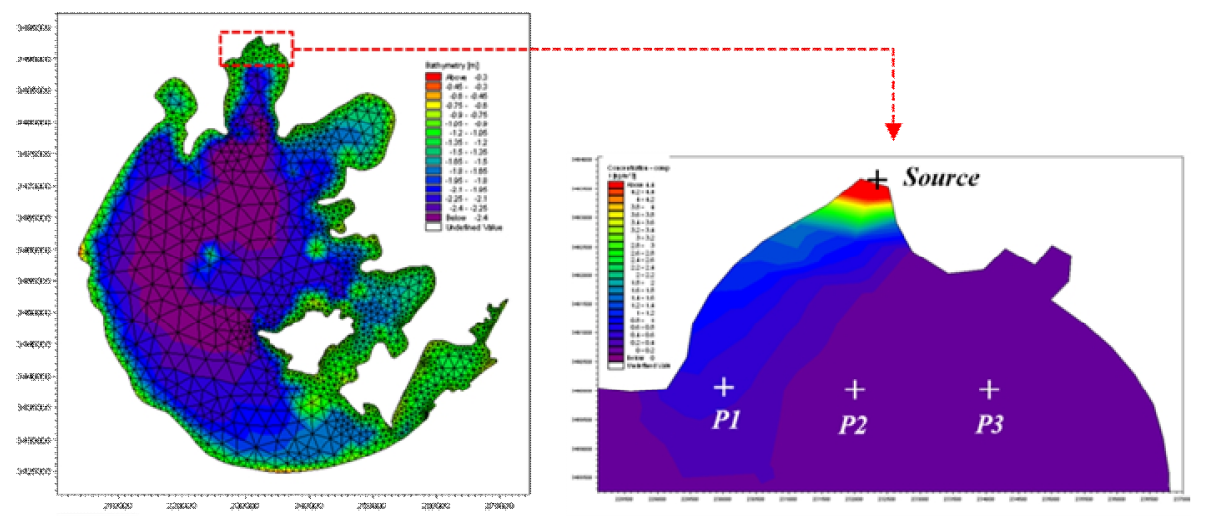

Fig.1. Horizontal mesh for the current and wave models (left) and locations for numerical transporting experiments (right)

River inflows were neglected as they had small effects on the short simulation of currents and waves. One open boundary was set as zero water levels at the outlet of Taipu River at the southeast of the lake. 
And zero velocity was given as initial condition, i.e. $u=0, v=0, w=0$. Constant wind conditions were used in the simulations. Two prevailing wind directions, southeast and northwest, were considered. And different wind speeds were set as $5 \mathrm{~m} / \mathrm{s}, 10 \mathrm{~m} / \mathrm{s}, 15 \mathrm{~m} / \mathrm{s}$, and $20 \mathrm{~m} / \mathrm{s}$, respectively.

Both the current and wave models were calibrated and validated before applications, according to the data reported by previous literatures ${ }^{[2,3]}$.

\section{Results and Discussions}

Effect of Wave on Current Speed. The simulation results of the wave-current coupled model were compared with those of the current model only (Fig. 2), and the results showed that the computed average velocities $(u$ and $v$ ) of each $\sigma$ layer under the same wind conditions had small differences. And both the two wind directions had similar results. The relative differences between the two from all the cases ranged from $-3.1 \%$ and $5.5 \%$, suggested that wave had limited influences on the computational results of currents under the given situations. Horizontally, the influence of wave on current was spatially heterogeneous, in which the current speed was increased at some lake areas and was reduced at other areas by waves.

Generally, when waves propagate into a following current, the individual components lengthen and their amplitudes are reduced, causing a drop in the surface elevation and spectral density ${ }^{[5]}$. And when waves propagate opposite the current, an increase in the current intensity is achieved near the mean water level, while a reduction is obtained for following waves and currents. It was also reported that waves with a shorter period have more intensive interaction with the ambient current, leading to a larger reduction in wave height and velocity magnitude around the water surface ${ }^{[6]}$.
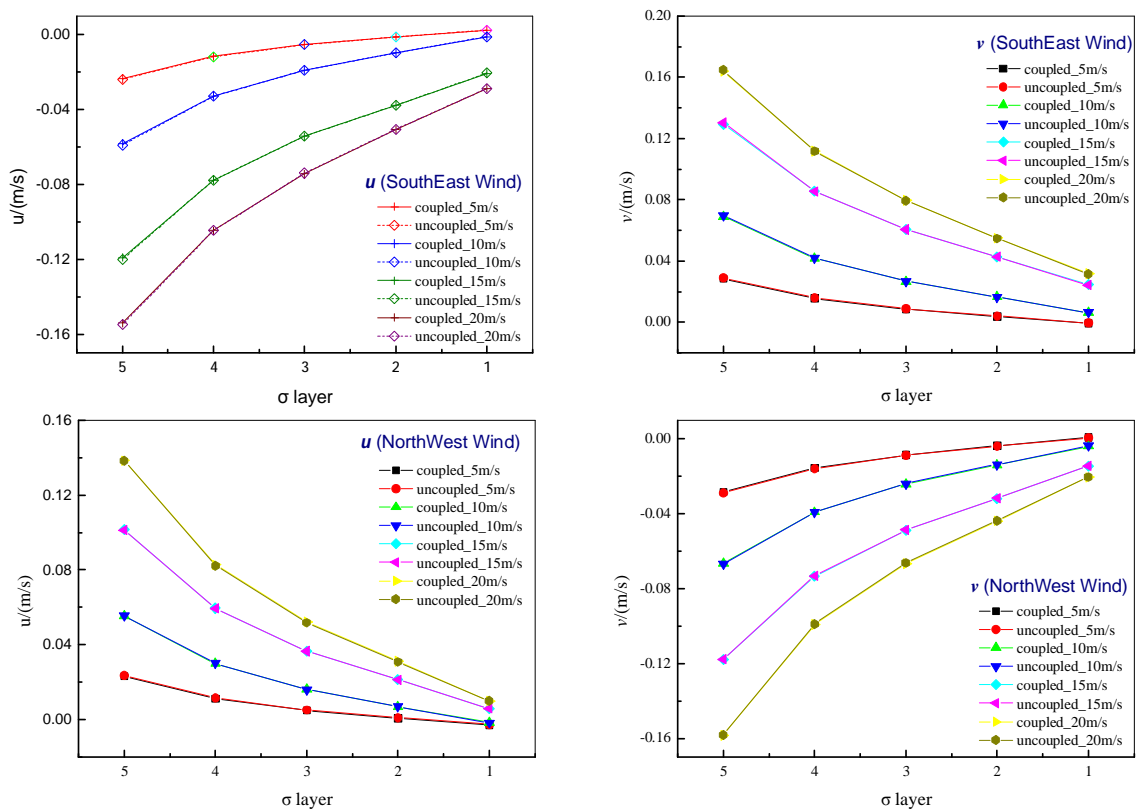

Fig. 2. Comparisons of the simulated current speeds between the coupled and current models

Effect of Current on Significant Wave Height. The simulated results of significant wave height from the wave model only, the coupled model, and their differences under two different wind conditions were shown in Fig. 3. It could be seen that the effect of current on significant wave height was also spatially heterogeneous, and varied with wind speed. The differences of significant wave height ranged from $-0.04 \mathrm{~m}$ to $0.05 \mathrm{~m}(-13.1 \%-14.0 \%)$ at the wind speed of $10 \mathrm{~m} / \mathrm{s}$, and from $-0.02 \mathrm{~m}-$ $0.06 \mathrm{~m}(-3.1 \%-7.8 \%)$ at $20 \mathrm{~m} / \mathrm{s}$, suggesting larger impact than that of wave to current. However, wind directions didn't affect much, as similar ranges of differences were found at the same wind speed for the two wind directions. 
In the wave model, the current field can affects the wave field in two ways: first, the wind input is modified by the current vector; second, the current vector modifies the wave action balance equation ${ }^{[7]}$. And the second was expected to be more important for wave spectrum tail ${ }^{[8]}$. In general, when waves travel with a following current, the induced wave height is smaller than that of wave-alone, and a great wave steepness could result in a larger reduction in wave height ${ }^{[6]}$.
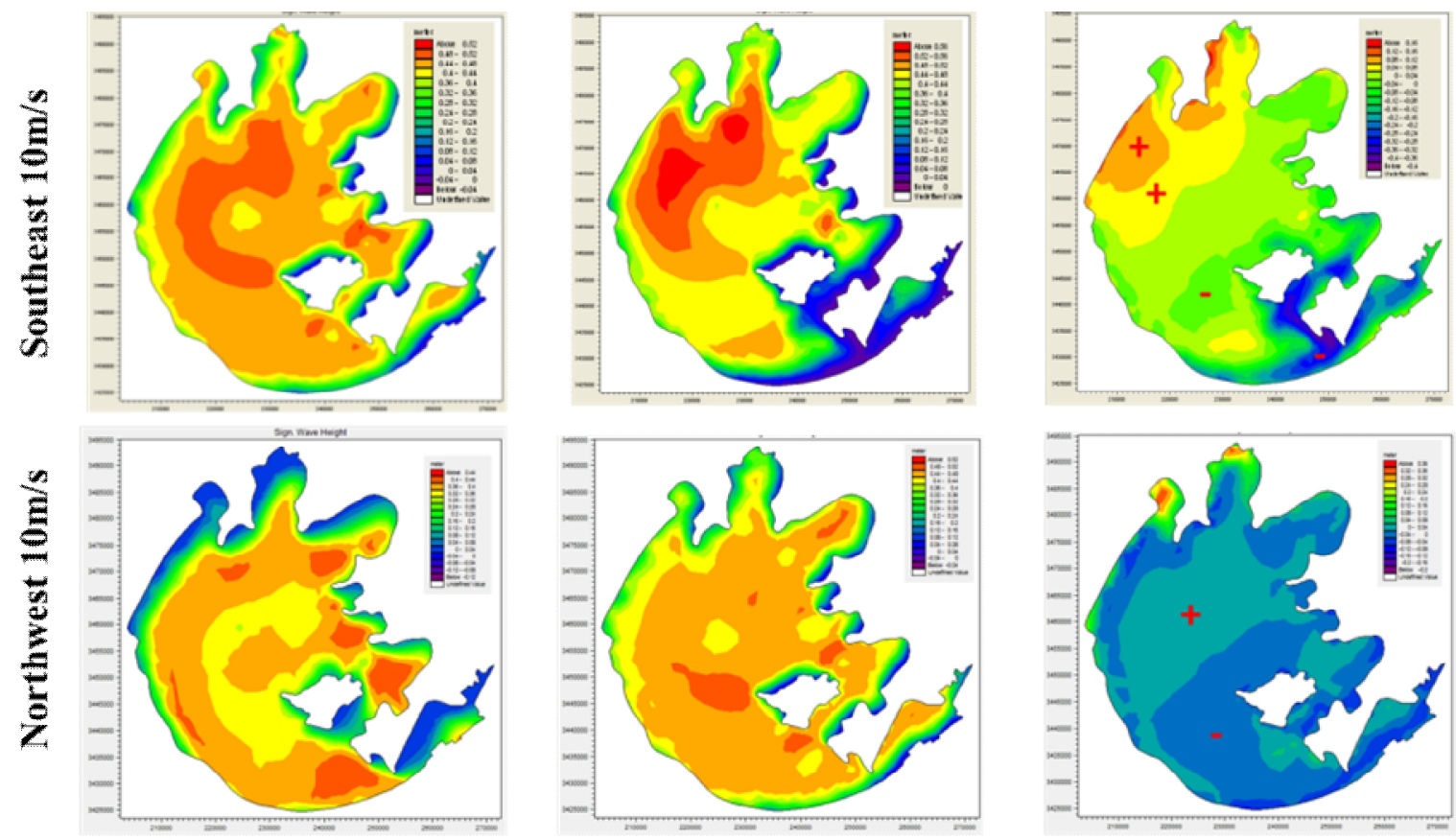

(a)

(b)

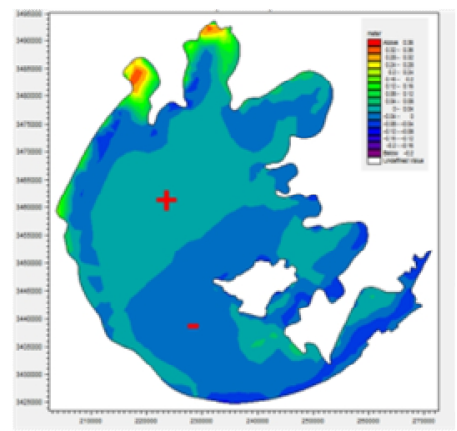

(c)

Fig.3. Simulated results of significant wave height from the wave model only (a), the coupled model (b), and their differences (c) under different wind conditions. The marks "+" and "-" in (c) stands for areas where significant wave height increased or reduced in the coupled model.

Wave-current Interaction on the Transport of Dissolved Materials. The concentrations of dissolved materials at the three probes (P1, P2, and P3) in the numerical experiments (southeast wind at $10 \mathrm{~m} / \mathrm{s}$ ) were shown in Fig. 4 . It could be seen that the computational results of the coupled model and the uncoupled current model were very similar, suggesting that the interaction had little effect on the simulation of dissolved matters in the water under the given situations.
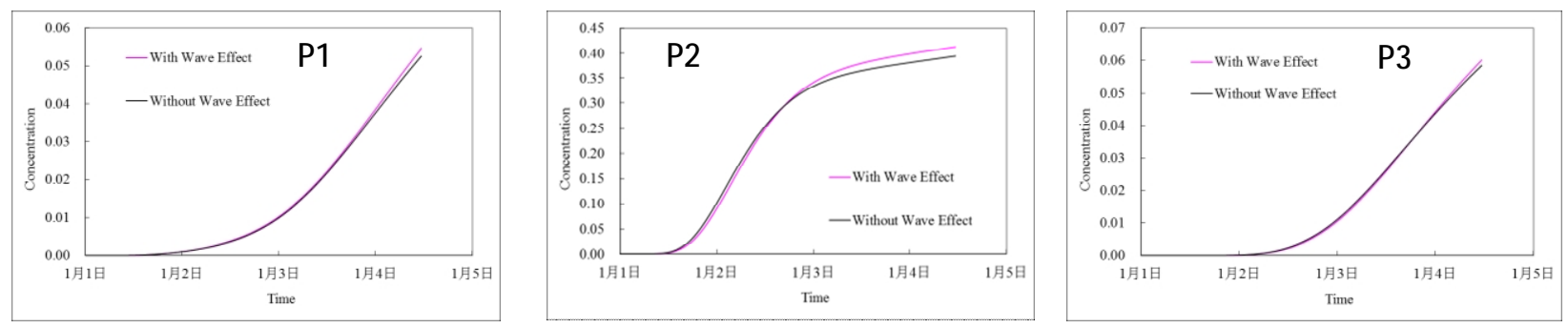

Fig. 4. Comparisons of simulated concentrations of dissolved materials at the three probes

Wave-current Interaction on the Transport of Particles. The concentrations of particles at the three probes (P1, P2, and P3) in the numerical experiments (southeast wind at $10 \mathrm{~m} / \mathrm{s}$ ) were shown in Fig.5. Compared to the results of dissolved matters, the results from the two models showed larger differences during the modelling period, suggesting that the wave-current interactions had more significant impact on the transport of particles than that of dissolved substances. As buoyant algae such as Microcystis aeruginosa has the potential to migrate in the water column exhibiting characteristics of particles, the interactions may need to be considered when modelling its transportation in the water. 

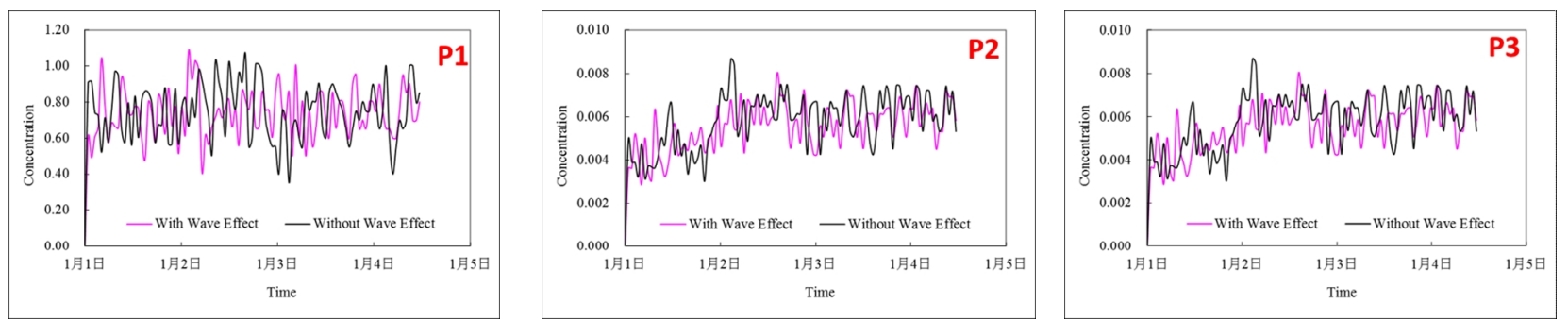

Fig. 5 Comparisons of simulated concentrations of particles at the three probes

\section{Conclusions}

The interaction of wind-induced wave and current and its potential effect on the transport of materials in Lake Taihu were studied through a wave-current coupled model. The influences between wave and current were spatially heterogeneous. Under the given conditions, wave had an influence of less than about $5 \%$ on the current speed, but current could lead to more than $10 \%$ difference of the computed significant wave height. And the wave-current interaction was found to have larger impact on particles than on dissolved substances in the surface water.

\section{Acknowledgements}

This work was financially supported by National Natural Science Foundation of China (11202217).

\section{References}

[1] B.Q. Qin, P.Z. Xu, Q.L. Wu, L.C. Luo and Y.L. Zhang: Hydrobiologia Vol. 581(1) (2007), p. 3-14

[2] L.C Luo and B.Q. Qin: Hydrology Vol. 23(3) (2003), p.1-4 (in Chinese)

[3] X.P. Liu: J. JIANGSU U: Nat. Sci. Ed., Vol. 30(1) (2009), p. 80-85 (in Chinese)

[4] DHI Water and Environment, in: DHI Software User Guide, Documentation and Reference Manual, MIKE 3: environmental hydraulics (2000)

[5] L. Rusu and C.G. Soares: Ocean. Eng. Vol. 38 (2011), p. 63-76

[6] J.S. Zhang, Y. Zhang, D.S. Jeng, P.L. Liu, and C. Zhang: Ocean. Eng. Vol. 75 (2014), p. 157-164

[7] A. Benetazzo, S. Carniel, M. Sclavo and A. Bergamasco: Ocean Model. Vol. 70 (2013), p. 152-165.

[8] Y. Fan, I. Ginis, T. Hara, C.W. Wright and E.J. Walsh: J. Phys. Oceanogr. Vol. 39 (2009), p. 2097-2116 